\title{
Conductance of single-atom platinum contacts: Voltage dependence of the conductance histogram
}

Nielsen, S.K.; Noat, Y.; Brandbyge, Mads; Smit, R.H.M.; Hansen, K.; Chen, L.Y.; Yanson, A.I.; Besenbacher, Flemming; Ruitenbeek, J.M.V

Published in:

Physical Review B Condensed Matter

Link to article, DOI:

10.1103/PhysRevB.67.245411

Publication date:

2003

Document Version

Publisher's PDF, also known as Version of record

Link back to DTU Orbit

Citation (APA):

Nielsen, S. K., Noat, Y., Brandbyge, M., Smit, R. H. M., Hansen, K., Chen, L. Y., Yanson, A. I., Besenbacher, F., \& Ruitenbeek, J. M. V. (2003). Conductance of single-atom platinum contacts: Voltage dependence of the conductance histogram. Physical Review B Condensed Matter, 67(24), 245411.

https://doi.org/10.1103/PhysRevB.67.245411

\section{General rights}

Copyright and moral rights for the publications made accessible in the public portal are retained by the authors and/or other copyright owners and it is a condition of accessing publications that users recognise and abide by the legal requirements associated with these rights.

- Users may download and print one copy of any publication from the public portal for the purpose of private study or research.

- You may not further distribute the material or use it for any profit-making activity or commercial gain

- You may freely distribute the URL identifying the publication in the public portal 


\title{
Conductance of single-atom platinum contacts: Voltage dependence of the conductance histogram
}

\author{
S. K. Nielsen, ${ }^{1}$ Y. Noat, ${ }^{2, *}$ M. Brandbyge, ${ }^{3}$ R. H. M. Smit, ${ }^{2}$ K. Hansen, ${ }^{1}$ L. Y. Chen, ${ }^{2}$ A. I. Yanson, ${ }^{2, \dagger}$ F. Besenbacher, ${ }^{1}$ \\ and J. M. van Ruitenbeek ${ }^{2, \$}$ \\ ${ }^{1}$ Interdisciplinary Nanoscience Center (iNANO), CAMP and Department of Physics and Astronomy, University of Aarhus, \\ DK-8000 Aarhus, Denmark \\ ${ }^{2}$ Kamerlingh Onnes Laboratory, Universiteit Leiden, Box 9504, 2300 RA Leiden, The Netherlands \\ ${ }^{3}$ Mikroelektronik Centret (MIC), Technical University of Denmark, Bldg. 345E, DK-2800 Lyngby, Denmark
}

(Received 10 December 2002; published 24 June 2003)

\begin{abstract}
The conductance of a single-atom contact is sensitive to the coupling of this contact atom to the atoms in the leads. Notably for the transition metals this gives rise to a considerable spread in the observed conductance values. The mean conductance value and spread can be obtained from the first peak in conductance histograms recorded from a large set of contact-breaking cycles. In contrast to the monovalent metals, this mean value for Pt depends strongly on the applied voltage bias and other experimental conditions and values ranging from about $1 G_{0}$ to $2.5 G_{0}\left(G_{0}=2 e^{2} / h\right)$ have been reported. We find that at low bias the first peak in the conductance histogram is centered around $1.5 G_{0}$. However, as the bias increases past $300 \mathrm{mV}$ the peak shifts to 1.8 $G_{0}$. Here we show that this bias dependence is due to a geometric effect where monatomic chains are replaced by single-atom contacts, where the former are destabilized by the electron current at high bias.
\end{abstract}

DOI: 10.1103/PhysRevB.67.245411

PACS number(s): 73.23.Ad, 73.63.Rt

The conductance of atomic-sized contacts (ASC's) is ballistic and can be written in terms of eigenchannels of the contact. In the limit of a single-atom contact the number of eigenchannels is governed by the number of valence orbitals of the atom. ${ }^{1}$ The simplest description, with a single conductance channel, applies for monovalent metals ( $\mathrm{Au}, \mathrm{Na}, \ldots)$, while for $s p$ metals three channels contribute, and for transition metals with open $d$ shells five channels contribute to the conductance. In general the transmission for each of the channels is smaller than 1 , but one often finds that the single channel for the monovalent metals is nearly perfectly transmitted giving a conductance of almost one conductance quantum, $G_{0}=2 e^{2} / h$, where $e$ is the electron charge and $h$ is Planck's constant. The five channels for the transition metals add up to a total conductance that sensitively depends on the coupling of the atom to its neighboring atoms in the leads and usually ranges between $1.5 G_{0}$ and $3.5 G_{0}$. This can be judged from the position and width of the first peak in so-called conductance histograms. For a recent review see Ref. 2.

The concept of conductance histograms was introduced to investigate possible conductance quantization in metallic contacts. ${ }^{3,4}$ The histograms are constructed from digitized traces of thousands of cycles of breaking (or making) ASC's. These traces are projected onto the conductance axis, and in this way preferred conductance values become visible as peaks. Only for monovalent metals, including the noble metals ${ }^{5,6}$ and the alkali metals, ${ }^{4,6}$ some form of quantization is observed as evidenced by prominent peaks in the histograms close to multiples of the conductance quantum. For most other metals there is no indication of quantization in the conductance histograms. ${ }^{2,6}$ There is usually a single peak at low conductance in the histograms that indicates the preferred conductance of the single-atom contacts. ${ }^{6}$ For most metals this first peak, when measuring in a clean environment, is fairly reproducible and insensitive to the level of the applied bias voltage. For $\mathrm{Au}$, which has been most studied, it has been shown that the conductance of the first peak is bias independent up to about $2 \mathrm{~V}$, which can be seen in the statistical average represented by conductance histograms measured for various bias voltages ${ }^{7}$ and from individual currentvoltage $(I-V)$ curves. ${ }^{8,9}$

$\mathrm{Pt}$ is a marked exception, and widely different results have been presented for this metal. The reported low bias position of the first peak varies between $1.0 G_{0}$ and 2.5 $G_{0} \cdot{ }^{3,10-14}$ Bias dependences of Pt conductance histograms have also been reported, ${ }^{14}$ where a low bias peak at $1.0 G_{0}$ was replaced by a peak at $1.7 G_{0}$ at higher biases. Recently it has been shown that at low bias the first peak is centered around $1.5 G_{0}$ (as in Fig. 1), and that a peak at $1.0 G_{0}$ can be caused by the presence of hydrogen molecules that act as the final bridge before the contact breaks. ${ }^{12}$

In this paper we investigate the bias dependence of $\mathrm{Pt}$ conductance histograms, compiled from thousands of ASC's

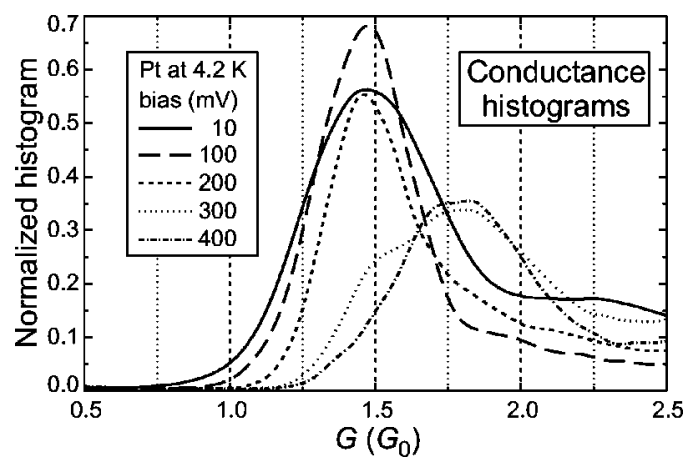

FIG. 1. Pt conductance histograms, each compiled from 3000 conductance traces of breaking ASC's recorded at $4.2 \mathrm{~K}$ under cryogenic vacuum in the MCBJ. The histograms are measured in succession on the same sample as the bias increases from 10 to $400 \mathrm{mV}$ and displays a clear dependence on the bias. (The samples are always made from a Pt wire with a purity of $99.999 \%$ and a diameter of $0.1 \mathrm{~mm}$.) The histograms have been normalized (Ref. 15). 
formed using the mechanically controllable break junction (MCBJ) (Refs. 2 and 10) at liquid-helium temperature (4.2 $\mathrm{K})$ in a cryogenic vacuum environment. The conductance measured is the linear conductance $G=I / V$. A small but significant shift in the position of the first histogram peak from $1.5 G_{0}$ to $1.8 G_{0}$ is observed when the bias increases past $300 \mathrm{mV}$. Contrary to what would have been expected from measured $I-V$ curves of $\mathrm{Pt}^{8}$ where the conductance decreases with voltage, the shift is towards higher conductance. This indicates that the shift is not caused by an electronic effect. We present evidence that the shift marks a geometric transition point where single-atom Pt contacts replace the monatomic chains known to exist for Pt. ${ }^{11}$

The bias dependence is illustrated by the conductance histograms shown in Fig. 1. For the curve recorded at a low bias of $10 \mathrm{mV}$ the first peak is found close to $1.5 G_{0}$. The conductance histogram recorded at $100 \mathrm{mV}$ is very similar to the one at $10 \mathrm{mV}$ with a broad peak centered around $1.5 G_{0}$. Increasing the bias further to $200 \mathrm{mV}$ only causes a slight decrease in the intensity, whereas the position remains unchanged. However, when the bias increases to $300 \mathrm{mV}$, the main part of the histogram peak switches to be centered around $1.8 G_{0}$ although a broad shoulder can still be seen around $1.5 G_{0}$. At $400 \mathrm{mV}$, the shoulder has disappeared and the peak center has completely moved from $1.5 G_{0}$ to 1.8 $G_{0}$. Also the peak intensity decreases considerably for the two high bias conductance histograms compared with those measured at lower bias. We have verified that the process is reversible, by lowering the bias back to zero observing the original histogram with a peak close to $1.5 G_{0}$ reemerge.

The peak shift to higher $G$ is very puzzling when compared to the Pt $I-V$ curves presented previously by Nielsen et al. ${ }^{8}$ It was shown that $I-V$ curves of Pt are markedly nonlinear but with a decreasing conductance as the voltage increases. The decrease in $G$ was found to be proportional to the voltage squared, resulting only in a limited decrease of the linear conductance at $300 \mathrm{mV}$ which amounts to about $-0.1 G_{0}$ at $500 \mathrm{mV}$. The important observation is, however, that the conductance always decreases and never increases with voltage, the opposite effect of the one observed with the conductance histograms presented in Fig. 1. This clearly indicates that the peak shift is not caused by an electronic effect.

Investigating other possible causes we turned to the formation of monatomic chains, which was first discovered for Au. ${ }^{16,17}$ Recently it was shown by Smit et al. that also Pt and Ir have this property. ${ }^{11}$ To demonstrate the formation of monatomic chains, Smit et al. recorded plateau-length histograms. It is important to emphasize the difference between plateau-length histograms ${ }^{11,17}$ and the conductance histograms used above. The peaks in the conductance histograms reveal the most probable conductance values obtained for breaking ASC's. A plateau-length histogram is constructed by measuring the lengths of the last conductance plateau, that corresponds to a conductor of a single atom in cross section, from thousands of conductance traces of breaking ASC's. By plotting the number of times a given length occurs, a histogram is obtained with peaks revealing the typical disruption lengths, at which the monatomic chains tend to

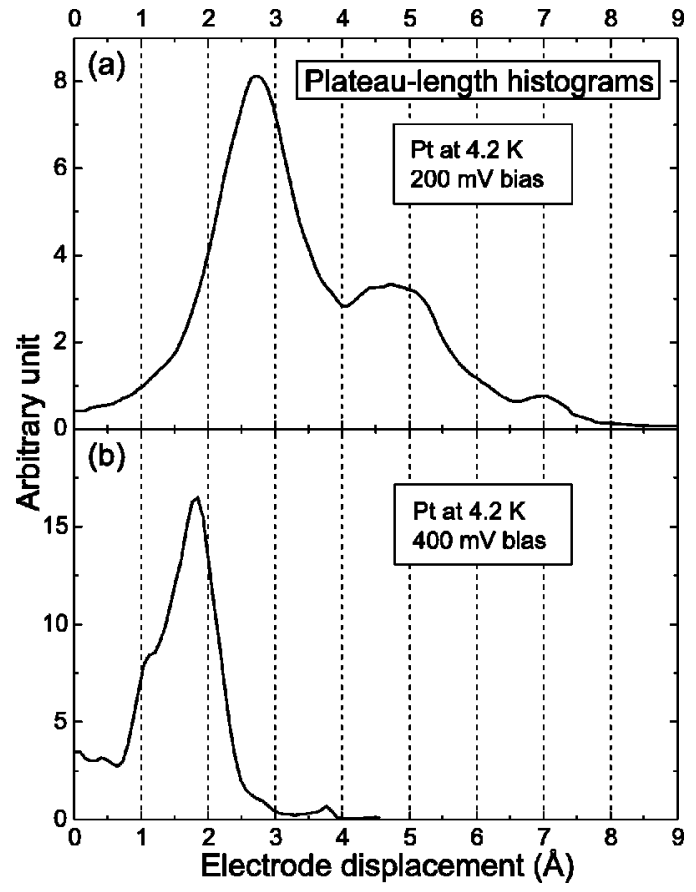

FIG. 2. Plateau-length histograms (Refs. 11 and 17) for Pt, each compiled from 2000 breaking ASC's. A bias of (a) $200 \mathrm{mV}$ and (b) $400 \mathrm{mV}$ is applied. The vertical axis gives a measure for the frequency with which a given chain length occurs.

break. The first peak reflects the length of the single-atom contact and peaks for longer wires indicate the formation of monatomic chains that break at $2,3,4, \ldots$ atoms in length.

In Fig. 2 we show two plateau-length histograms recorded at biases of (a) $200 \mathrm{mV}$ and (b) $400 \mathrm{mV}$, respectively. At 200 $\mathrm{mV}$ bias, the plateau-length histogram is very similar to the one presented by Smit et al. at $10 \mathrm{mV}$ bias. ${ }^{11}$ Three clear peaks are visible, the first corresponding to a single-atom contact (a "one atom chain"), and the next peaks represent chains with two and three atoms, respectively. Thus we conclude that monatomic chains still form at a bias as high as $200 \mathrm{mV}$. The three peaks in Fig. 2(a) are centered at 2.7, 4.8, and 7.0 $\AA$, respectively. The distances between them are thus 2.1-2.2 $\AA$, similar to the $2.3 \AA$ found by Smit et al. at 10 $\mathrm{mV}$ bias, ${ }^{11}$ within the accuracy of $\sim 10 \%$ in our length calibration. The position of the first peak in Fig. 2(a) differs from this peak-to-peak distance as can be expected. It reflects the elastic stretching of the banks and the bonds to a single bridging atom at the verge of breaking.

The presence of only one single peak in the $400-\mathrm{mV}$ plateau-length histogram of Fig. 2(b), centered at $1.9 \AA$, proves that chains no longer form at this high bias. The peak does not coincide with the corresponding first peak in the $200-\mathrm{mV}$ plateau-length histogram. This difference is most likely due to the higher bias causing the single-atom contact to break at a smaller strain than at low bias (see below).

From the plateau-length histograms we find that the formation of atomic chains is inhibited above the bias voltage for which also the shift in the first peak in the conductance histogram is observed $(300 \mathrm{mV})$. This suggests that the chain formation affects the peak position in the conductance histograms. To test this we recorded conductance histograms for 


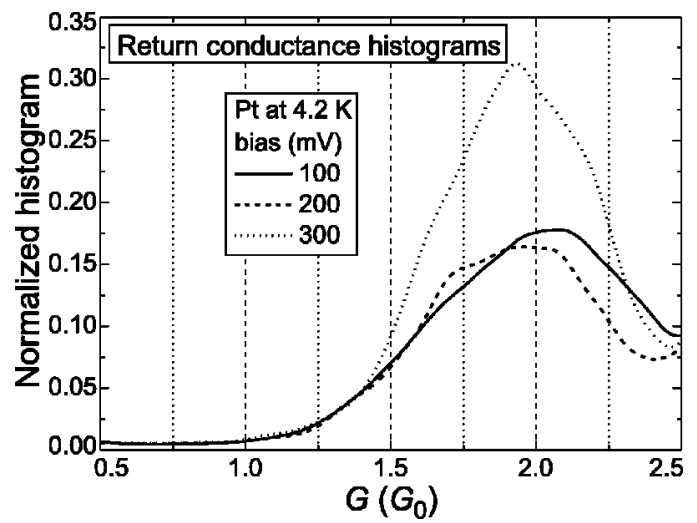

FIG. 3. Return conductance histograms of Pt. Each histogram is compiled from 2000 conductance traces and is measured in succession on the same sample while the bias increases from 100 to 300 $\mathrm{mV}$ in 100-mV steps. The measurements are performed by forming the ASC's with the MCBJ. The histograms have been normalized (Ref. 15).

curves measured while returning to contact from the vacuum tunneling regime, which we will refer to as return histograms. The conductance histograms presented previously have all been obtained from the conductance traces of breaking ASC's. When breaking the contacts, monatomic chain formation may occur. Instead, when we obtain the ASC's by returning the electrodes back into contact, chains cannot form. Measuring the conductance traces while forming ASC's, results in the return histograms presented in Fig. 3. From these return histograms it is clear that the first peak is centered close to $2 G_{0}$, independent of bias. It thus seems that the peak shift occurs when the chain formation is inhibited, since the peak is located at an even higher conductance, independent of bias, when no chains can form.

For $\mathrm{Au}$ the conductance of a single-atom contact is indistinguishable from that of monatomic chains and we had expected a similar result for $\mathrm{Pt}$, but the present results suggest otherwise. To get further insight into these findings, we have used the TRANSIESTA program ${ }^{18}$ to calculate the conductance and eigenchannel transmissions of single-atom $\mathrm{Au}$ and $\mathrm{Pt}$ contacts [inset of Fig. 4(a)] as the atom-electrode distance increases. The results are shown in Fig. 4. The method is based on density functional theory and takes the voltage bias and current explicitly into account in the self-consistent calculation of electronic density and potential. Our calculations show that, contrary to Au for which the conductance remains stable around $1 G_{0}$, single-atom Pt contacts display a strong variation in the zero-bias conductance with a decrease from $2.1 G_{0}$ to $1.1 G_{0}$ as the distance increases from 2.65 to 3.5 $\AA$. This variation is in accordance with the reported broad histogram peak centered around $(1.5-2) G_{0}$ for $\mathrm{Pt}^{11-13}$ These results can help explain the behavior leading to the three types of histograms presented above.

The histogram with a peak at $2 G_{0}$ (Fig. 3) is produced by single-atom contacts. In this case the electrodes are moved towards each other such that the atom-electrode distance is reduced to a minimum. The peak at $1.8 G_{0}$ in the high-bias histograms (Fig. 1) also results from single-atom contacts, but in this case the breaking of the wire leads to a larger
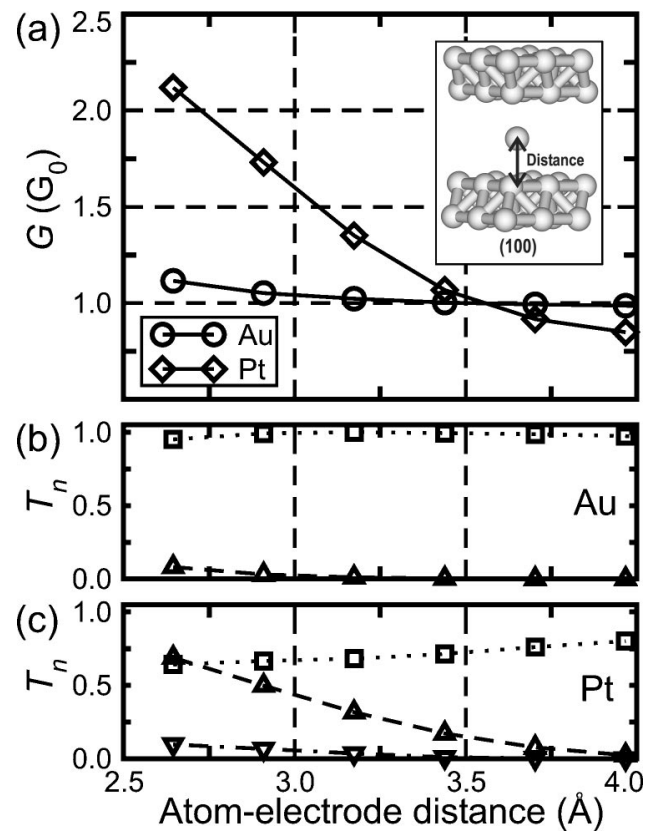

FIG. 4. (a) Calculated conductance $G$ vs atom-electrode distance at zero voltage for a symmetric single-atom $\mathrm{Au}$ or Pt contact configuration consisting of a single atom between two $\mathrm{Au}$ or $\mathrm{Pt}(100)$ surfaces (inset). Note that the contacts in practice will become unstable beyond a distance of about $3 \AA$. (b) and (c). The conductance decomposed into eigenchannel transmissions for $\mathrm{Au}$ and $\mathrm{Pt}$. The dotted line corresponds to the nondegenerate channel consisting of $s$ and $d_{z^{2}}$ orbitals, the dashed line corresponds to the two degenerate channels with the $d_{z x}$ and $d_{y z}$ orbitals, while the dot-dashed corresponds to the single channel with $d_{x^{2}-y^{2}}$.

atom-electrode distance, and thus a lower conductance (Fig. 4). The peak at $1.5 G_{0}$ in the low-bias histograms of Fig. 1 is due to the frequent occurrence of atomic chains, which are only stable for sufficiently low bias voltages, below $300 \mathrm{mV}$. The lower average conductance for atomic chains is suggested by the physics described in Fig. 4. As the single atom is positioned farther away from the banks the overlap of its orbitals with those in the electrodes is reduced leading to the observed decrease in the conductance. For monatomic chains the number of states overlapping with the central atoms in the chains is also reduced with respect to the single-atom case, leading to a similar reduction of the conductance. The suppression of chain formation at higher bias is partly due current-induced embrittlement, but mainly results from heating of the atomic degrees of freedom by the current. ${ }^{19,20}$

We can now attempt to classify the various results found in the literature. A first peak in the conductance histogram close to $1 G_{0}$ was found in room-temperature experiments under ultrahigh vacuum, ${ }^{3}$ or under an atmosphere of $\mathrm{N}_{2}$ $+5 \% \mathrm{H}_{2}$ gas. $^{14}$ For the latter experiment the peak was seen to move to $1.7 G_{0}$ at elevated bias. For all experiments performed under cryogenic vacuum and at a voltage bias below $100 \mathrm{mV}$ (Refs. 10-13 and this work) the first peak is found at $(1.5-1.6) G_{0}$. However, when hydrogen is intentionally introduced into the vacuum system a peak near $1 G_{0}$ appears, which has been interpreted as being due to the formation of 
a conducting hydrogen bridge forming the last contact. ${ }^{12}$ Assuming this mechanism is still effective at room temperature the results by Yuki et al. ${ }^{14}$ find a natural explanation. Trace amounts of hydrogen in the UHV system at room temperature may similarly explain the observation by Olesen et al. ${ }^{3}$

The remaining variation in the position of the first peak in the $\mathrm{Pt}$ conductance histograms can be attributed to a bias dependence of the formation of monatomic chains. The shift of the first peak in the Pt conductance histogram from $1.5 G_{0}$ to $1.8 G_{0}$ when the bias increases past $300 \mathrm{mV}$, marks a geometric transition point where monatomic chains are replaced by single-atom contacts. ${ }^{21}$

We acknowledge financial support from The Center for Atomic-scale Materials Physics (CAMP) sponsored by the Danish National Research Foundation and from the EU network "Bottom up Nanomachines" (BUN). M.B. acknowledges support from the Danish Natural Science Research Council. Y.N. has been supported by the European Community under Contract No. HPMF-CT-1999-00196.
*Present address: Groupe de Physique des Solides, Campus Jussieu tour 23, 2 Place Jussieu, 75251 Paris cedex 05, France.

†Present address: Dept. of Physics, 510 Clark Hall, Cornell University, Ithaca, NY 14853.

Corresponding author. Electronic address: ruitenbe@ Phys.LeidenUniv.nl

${ }^{1}$ E. Scheer, N. Agraï, J.C. Cuevas, A. Levy Yeyati, B. Ludoph, A. Martn-Rodero, G. Rubio Bollinger, J.M. van Ruitenbeek, and C. Urbina, Nature (London) 394, 154 (1998).

${ }^{2}$ N. Agraï, A. Levy Yeyati, and J.M. van Ruitenbeek, Phys. Rep. 377, 81 (2003).

${ }^{3}$ L. Olesen, E. Lægsgaard, I. Stensgaard, F. Besenbacher, J. Schiøtz, P. Stoltze, K.W. Jacobsen, and J.K. Nørskov, Phys. Rev. Lett. 74, 2147 (1995).

${ }^{4}$ J.M. Krans, J.M. van Ruitenbeek, V.V. Fisun, I.K. Yanson, and L.J. de Jongh, Nature (London) 375, 767 (1995).

${ }^{5}$ M. Brandbyge, J. Schiøtz, M.R. Sørensen, P. Stoltze, K.W. Jacobsen, J.K. Nørskov, L. Olesen, E. Lægsgaard, I. Stensgaard, and F. Besenbacher, Phys. Rev. B 52, 8499 (1995).

${ }^{6}$ B. Ludoph and J.M. van Ruitenbeek, Phys. Rev. B 61, 2273 (2000).

${ }^{7}$ H. Yasuda and A. Sakai, Phys. Rev. B 56, 1069 (1997); K. Yuki, A. Enomoto, and A. Sakai, Appl. Surf. Sci. 169-170, 489 (2001)

${ }^{8}$ S.K. Nielsen, M. Brandbyge, K. Hansen, K. Stokbro, J.M. van Ruitenbeek, and F. Besenbacher, Phys. Rev. Lett. 89, 066804 (2002).

${ }^{9}$ K. Hansen, S.K. Nielsen, M. Brandbyge, E. Lgsgaard, I. Stensgaard, and F. Besenbacher, Appl. Phys. Lett. 77, 708 (2000).
${ }^{10}$ J.M. Krans, C.J. Muller, I.K. Yanson, T.C.M. Govaert, R. Hesper, and J.M. van Ruitenbeek, Phys. Rev. B 48, 14721 (1993).

${ }^{11}$ R.H.M. Smit, C. Untiedt, A.I. Yanson, and J.M. van Ruitenbeek, Phys. Rev. Lett. 87, 266102 (2001).

${ }^{12}$ R.H.M. Smit, Y. Noat, C. Untiedt, N.D. Lang, M.C. van Hemert, and J.M. van Ruitenbeek, Nature (London) 419, 906 (2002).

${ }^{13}$ C. Sirvent, J.G. Rodrigo, S. Vieira, L. Jurczyszyn, N. Mingo, and F. Flores, Phys. Rev. B 53, 16086 (1996).

${ }^{14}$ K. Yuki, S. Kurokawa, and A. Sakai, Jpn. J. Appl. Phys., Part 1 39, 4593 (2000).

${ }^{15}$ The conductance histograms in Figs. 1 and 3 have been normalized by the area under the curve in the conductance range from 0 to $10 G_{0}$. Since the figures show a zoom to $0.5-2.5 G_{0}$ the area in the figures are not the same.

${ }^{16}$ H. Ohnishi, Y. Kondo, and K. Takayanagi, Nature (London) 395, 780 (1998).

${ }^{17}$ A.I. Yanson, G.R. Bollinger, H.E. van den Brom, N. Agraït, and J.M. van Ruitenbeek, Nature (London) 395, 783 (1998).

${ }^{18}$ M. Brandbyge, J.-L. Mozos, P. Ordejón, J. Taylor, and K. Stokbro, Phys. Rev. B 65, 165401 (2002); J.M. Soler, E. Artacho, J.D. Gale, A. García, J. Junquera, P. Ordejón, and D. SánchezPortal, J. Phys.: Condens. Matter 14, 2745 (2002).

${ }^{19}$ T.N. Todorov, J. Hoekstra, and A.P. Sutton, Phys. Rev. Lett. 86, 3606 (2001).

${ }^{20}$ R. H. M. Smit, Ph.D. thesis, Universiteit Leiden, The Netherlands, 2003.

${ }^{21}$ For further details, see S. K. Nielsen, Ph.D. thesis, University of Aarhus, Denmark, 2002. 